\title{
Statistical Recognition Method of Binary BCH Code
}

\author{
Jiafeng Wang, Yang Yue, Jun Yao \\ Institute of Electronic Engineering in China Academy of Engineering Physics, \\ Mianyang, China \\ E-mail: hugeghost@hotmail.com \\ Received October 30, 2010; revised January 5, 2010; accepted January 6, 2011
}

\begin{abstract}
In this paper, a statistical recognition method of the binary BCH code is proposed. The method is applied to both primitive and non-primitive binary $\mathrm{BCH}$ code. The block length is first recognized based on the cyclic feature under the condition of the frame length known. And then candidate polynomials are achieved which meet the restrictions. Among the candidate polynomials, the most optimal polynomial is selected based on the minimum rule of the weights sum of the syndromes. Finally, the best polynomial was factorized to get the generator polynomial recognized. Simulation results show that the method has strong capability of anti-random bit error. Besides, the algorithm proposed is very simple, so it is very practical for hardware implementation.
\end{abstract}

Keywords: Binary BCH Code, Blind Recognition, Code Length, Generator Polynomial

\section{Introduction}

With the development of the Communication Countermeasures, the methods of the communication countermeasures have transferred from the signal level to the information level. The blind recognition of channel coding is the foundation of getting the message, therefore has become the key technology in the area of the Communication Countermeasures and gets more and more attention. Besides, the blind recognition of channel coding also has important applications in cooperative areas such as the intelligent communication.

The linear channel coding has two categories: block code and convolutional code. We can see that most researches focus on the blind recognition of convolutional code [1-2], while literature about the blind recognition of block code is rare. Literature [3] gives a blind recognition method of the block code with low encoding rate, its essential principle is to resolve equations to recognize the syndrome matrix, and then get the generator matrix. Literature [4] discusses a method of blind recognition of primitive RS code, its essential principle is using reduced row echelon form of the matrix (RREF), fault-tolerant matrix decomposing (FTMD) and Galois field Fourier transform (GFFT) to recognize encoding parameters and syndrome matrixes. Both methods need matrix computations on the finite field, when code length is long, the EMS memory needed will be very large, so it is not ap- plicable to hardware implementation. At the same time, the error code capability is not ideal too.

In this paper we study the binary $\mathrm{BCH}$ code which is an important subclass of cyclic codes. According to the circular feature, we proposed a simple statistical recognition method under the condition that the frame length is known. The method has strong anti-error capability. Besides, there is no need for matrix computation; therefore it is suitable for hardware implementation.

The reset of this paper is organized as follows: Section 1 introduces the basic feature of binary $\mathrm{BCH}$ code; Section 2 discusses the recognition method of the block length; Section 3 discusses the method of recognition of the generator polynomial; Section 4 observes the recognition performance of this algorithm through simulation; finally, the paper is briefly summarized.

\section{Binary BCH Code}

Here we simply introduce the features of binary $\mathrm{BCH}$ code. The definition of binary $\mathrm{BCH}$ code and the details of encoding and decoding are discussed in literature [5].

Consider $(n, k) \mathrm{BCH}$ code defined on $\mathrm{GF}(2), n$ is the block length and $k$ is the message length. Let $m=\left(m_{0}, m_{1}, \cdots, m_{k-1}\right)$ be the message word before encoding, $c=\left(c_{0}, c_{1}, \cdots, c_{n-1}\right)$ be the code word after encoding, because it is the cyclic code, the message word 
and code word each has a corresponding message polynomial and code polynomial defined on $\mathrm{GF}(2)$, as follows

$$
\begin{gathered}
m(x)=m_{0} x^{k-1}+m_{1} x^{k-2}+\cdots+m_{k-2} x+m_{k-1} \\
c(x)=c_{0} x^{n-1}+c_{1} x^{k-2}+\cdots+c_{k-2} x+c_{n-1}
\end{gathered}
$$

From literature [5], we know that the $(n, k)$ binary $\mathrm{BCH}$ code has following features,

1) Block length $n$ equals $2^{m}-1$ or is a factor of $2^{m}-1$, where $m \geq 3$. If $n$ equals $2^{m}-1$, then it is primitive binary $\mathrm{BCH}$ code; otherwise, it is non- primitive one.

2) The code word $c$ satisfies the circular feature, that is, after circular shifting the code word $c$ for $j$ times, get the code word

$$
c^{\prime}=\left(c_{j-1}, c_{j}, \cdots, c_{k-1}, c_{0}, \cdots, c_{j-3}, c_{j-2}\right)
$$

and $c^{\prime}$ also belongs to $(n, k)$ binary $\mathrm{BCH}$ code set.

3) $m(x)$ and $c(x)$ satisfy following relationship,

$$
c(x)=m(x) g(x)
$$

where $g(x)$ is the generator polynomial defined on GF (2),

$$
g(x)=x^{n-k}+g_{1} x^{n-k-1}+\cdots+g_{n-k-1} x+1
$$

4) There exists syndrome polynomial $h(x)$ defined on $\operatorname{GF}(2)$,

$$
h(x)=x^{k}+h_{1} x^{k-1}+\cdots+h_{k-1} x+1
$$

which satisfies following relationships,

$$
\begin{gathered}
h(x) c(x)=0 \bmod \left(x^{n}+1\right) \\
h(x) g(x)=x^{n}+1
\end{gathered}
$$

5) $g(x)$ is irreducible.

\section{Recognition of Block Length}

The recognition of the binary $(n, k) \mathrm{BCH}$ code is to recognize three parameters: the block length $n$, the syndrome word length $k$, and the generator polynomial $g(x)$. But we can see from formula (4) that the order of $g(x)$ is $n-k$, then as long as the block length $n$ and the generator polynomial $g(x)$ are recognized, the value of $k$ can be ascertained. Then only the block length $n$ and the generator polynomial $g(x)$ need to be recognized. In this section we introduce the recognition of block length $n$, and in next section we introduce the recognition of generator polynomial $g(x)$.

The recognition is based on the assumption that frame length $f_{l}$ is known. This assumption is rational, be- cause usually in practice, frame head is not encoded, so it is easy to get.

Because $f_{l}$ is known, and there exists at least two code words in one frame, so we could get following conclusions:

1) $n \in\left[3,\left\lfloor\frac{f_{l}}{2}\right\rfloor\right]$, where $\lfloor\cdot\rfloor$ means floor function.

2) $f_{l}$ is divisible by $n$, that is, $n$ is a factor of $f_{l}$.

The factor numbers of $f_{l}$ in the rang $\left[3,\left[\frac{f_{l}}{2}\right\rceil\right]$ may not be only one, assume $i$ is a factor of $f_{l}$ and then there are two situations,

1) $i=n$

Under this situation, if we take $i$ as the block length, we could get $N_{i}$ code words. Assume $c_{p}(x)$ is the code polynomial of the $p$ th code word, then through left circular shifting for $j$ times we get $j$ code polynomials $c_{p 1}(x), c_{p 2}(x), \cdots, c_{p j}(x)$, where $1 \leq j \leq i-1$.

Because the binary $\mathrm{BCH}$ code satisfy the circular feature, if there is no error code in $c_{p}$, then the code words corresponding to $c_{p 1}(x), c_{p 2}(x), \cdots, c_{p j}(x)$ and the code word corresponding to $c_{p}(x)$ belong to the same $(n, k)$ binary $\mathrm{BCH}$ set, so their generator polynomials are the same. According to formula (3) which is the relationship between the code polynomial and the generator polynomial, we know there is a common factor for $c_{p}(x), c_{p 1}(x), c_{p 2}(x), \cdots, c_{p j}(x)$. Let $c_{p 0}(x)=c_{p}(x)$, then the following relationship exists,

$$
\operatorname{gcd}\left[c_{p 0}(x), c_{p 1}(x), \cdots, c_{p j}(x)\right] \neq 1 \quad 1 \leq j \leq i-1
$$

We call the code word that satisfies fomula (8) as a valid code word. Assume there are $N_{i c}$ valid code words among the $N_{i}$ code words. Obviously, when there is no error code, $N_{i c}=N_{i}$, that is, the percentage of the valid code words of all the code words $f_{i c}$ is,

$$
f_{i c}=\frac{N_{i c}}{N_{i}}=1
$$

2) $i \neq n$

Under this situation, if we take $i$ as the block length, the blocking is wrong. Assume we get $N_{i}$ code words, there exist code words that do not satisfy formula (8) inevitably, so $N_{i c}<N_{i}$, that is

$$
f_{i c}=\frac{N_{i c}}{N_{i}}<1
$$

Above all, under the condition that no error code exists, if take formula (8) as the rule to judge if the code word is valid or not, then for all the possible block lengths, when $i=n$, the percentage of valid code words is $f_{i c}=1$; when $i \neq n$, the percentage of valid code words is $f_{i c}<1$. Of course, when there are error codes 
exist, even if $i=n, f_{\text {ic }}$ could be less than 1. But we can predict that $f_{i c}$ should get the maximum when $i=n$.

In addition, according to feature (1), $n$ is odd, so we could get the recognition formula of the block length $n$,

$$
\begin{aligned}
n= & \arg \max _{i}\left(f_{\text {ic }}\right) \\
& i \in\left[3,\left\lfloor f_{l} / 2\right]\right] \\
& \operatorname{rem}(i, 2)=1 \\
& \operatorname{rem}\left(f_{l}, i\right)=0
\end{aligned}
$$

where $\max (\cdot)$ means the maximum operation; $\operatorname{rem}\left(f_{l}, i\right)$ means the remainder of $f_{l}$ divided by $i$. The rule to judge the validity of the code word is formula (8). Then we can get the recognition process of binary $\mathrm{BCH}$ code block length $n$ :

1) Let $i=3$ and initialize the value of $j$;

2) If $i$ can not divide $f_{l}$, then turn to (6);

3) Let $i$ be the block length and get $N_{i}$ code words;

4) According to formula (8), calculate the number of valid code words $N_{i c}$;

5) Compute $f_{i c}=\frac{N_{i c}}{N_{i}}$ and save;

6) $i=i+2$;

7) If $i \leq\left\lceil f_{l} / 2\right\rceil$, turn to (2);

8) Compare all the saved $f_{i c}$, the estimation of $n$ is $i$ that made $f_{i c}$ has maximum value;

9) The recognition process is over.

We can see from step (11) and step (8), the recognition capability of block length $n$ is relevant to $N_{i}$ and $j$, the more the value of $N_{i}$ and $j$, the better the recognition capability at the first glance; but large values mean that the length of the received code stream series is also large, and the computation complexity will increase, so we must consider this tradeoff according to the practical situations.

\section{Recognition of Generator Polynomial}

In this section, we discuss how to recognize the generator polynomial $g(x)$ under the condition of the block length $n$ is rightly recognized.

Assume we receive $N$ code words, $c_{p}(x)$ is the code polynomial for the $p$ th code word, and assume that there is no error code. According to section II, left circular shift $c_{p}(x)$ to get $n-1$ code polynomials $c_{p 1}(x)$, $c_{p 2}(x), \cdots, c_{p n-1}(x)$, and the generator polynomials of these $n-1$ code polynomials and $c_{p}(x)$ are the same. In other words, the generator polynomial $g(x)$ is a common factor of $c_{p}(x), c_{p 1}(x), c_{p 2}(x), \cdots$, $c_{p n-1}(x)$. Also let $c_{p 0}(x)=c_{p}(x)$, and

$$
f_{p}(x)=\operatorname{gcd}\left[c_{p 0}(x), c_{p 1}(x), \cdots, c_{p j}(x)\right]
$$

Then $f_{p}(x)$ is the multiple of $g(x)$.

From the received $N$ code words, we can get $M$ ( $1 \leq M \leq N)$ different polynomials according to formula (12), consider there are error codes, these $M$ polynomials belong to one of the four situations below:

1) Equal 1;

2) Not equals 1 , but not equals $g(x)$, also not the multiple formula of $g(x)$;

3) Equal $g(x)$;

4) Equal the multiple formula of $g(x)$.

Situations (1) and (2) illustrate there are error codes exist. Situations (3) and (4) illustrate there are no error codes exist in code words, or the error codes constitute another code words in the same sub code set. We can get the generator polynomial from the $M$ candidate polynomials following three steps below.

Step 1. According to the constraint conditions of $g(x)$ satisfied, remove the polynomials that do not satisfy the conditions

According to the feature of the binary circular code, $g(x)$ needs to satisfy the following restrictions [5],

1) $g(x) \neq 1$

2) $g(x) \neq x^{q}+1$, where $q$ is the positive integer and $1 \leq q<n$

3) $g(x)$ can divide $x^{n}+1$

According to these three restrictions to remove the polynomials under situations (1) and (2).

Step 2. According to the minimum rule of the weights sum of the syndromes choose the most optimal polynomial

Assume after the step 1 there are $L$ candidate polynomials remain, and $g_{i}(x) \quad(i=1,2, \cdots, L)$ is the $i$ th polynomial, and its corresponding syndrome polynomial is,

$$
h_{i}(x)=\left(x^{n}+1\right) / g_{i}(x)
$$

The $j$ th syndrome of the code word is,

$$
r_{i j}=\left[h_{i}(x) c_{j}(x)\right] \bmod \left(x^{n}+1\right)
$$

The code weight is,

$$
w_{i j}=\text { weight }\left(r_{i j}\right)
$$

For all the received $N$ code word compute the syndrome code weight,

$$
w_{i}=\sum_{j=1}^{N} w_{i j} \quad i=1,2, \cdots, L
$$

The candidate polynomial made $w_{i}$ minimum is the most optimal polynomial, denotes as $g_{b}(x)$,

$$
g_{b}(x)=\underset{g_{i}(x), i=1,2, \cdots, L}{\arg \min }\left(w_{i}\right)
$$

Step 3. From the most optimal polynomial $g_{b}(x)$ to get the estimated generator polynomial $g_{0}(x)$. 
When the reorganization is correct, the most optimal polynomial $g_{b}(x)$ is not necessarily equals $g(x)$, but it is definitely the multiple of $g(x)$, so further steps are needed using $g_{b}(x)$ to estimate the generator polynomial.

Firstly according to the rules in [6], judge whether $g_{b}(x)$ is reducible or not; if it is irreducible, then

$$
g_{0}(x)=g_{b}(x)
$$

If it is reducible, get all the irreducible factor of $v g_{b}(x)$ that satisfy step 1 , then follow step 2 to get the most optimal polynomial as the estimated generator polynomial $g_{0}(x)$. The method of factorization can indirectly get from the rules in [6], we do not comment on this because the limitation of space.

From the above recognition process, theoretically, as long as there is a code word without error code, it is of very high possibility to correctly recognize the generator polynomial, then this recognition method has strong anti-error capability.

\section{Simulation}

The simulation has three steps: first, we simulate for the recognition capability of the block length; second, we simulate for the recognition capability of the generator polynomial; third, according to the first two steps we get the total recognition capability.

In the simulation of the recognition capability, we run the simulation 1000 times for $(15,11)$ primitive binary $\mathrm{BCH}$ code and $(21,12)$ non-primitive binary $\mathrm{BCH}$ code respectively to get the statistical correct recognition rate. The generator polynomial of $(15,11)$ primitive binary $\mathrm{BCH}$ code is $g_{1}(x)=x^{4}+x+1$; the generator polynomial of $(21,12)$ non-primitive binary $\mathrm{BCH}$ code is $g_{1}(x)=x^{9}+x^{3}+1$; assume that there are five code words in one frame, and we choose the circular shifting time as 1 for recognizing the block length.

\subsection{Simulation Results}

The recognition simulations are done according to the method described in Sections 2 and 3. The results are shown in Figures 1-6. The total recognition capability is computed according to the block length recognition capability and generator length recognition capability, the computation formula is as follows,

$$
p=p_{1} \cdot p_{2}
$$

where $p$ is the total correct recognition rate, $p_{1}$ is the correct recognition rate of block length, $p_{2}$ is the correct recognition rate of generator polynomial.

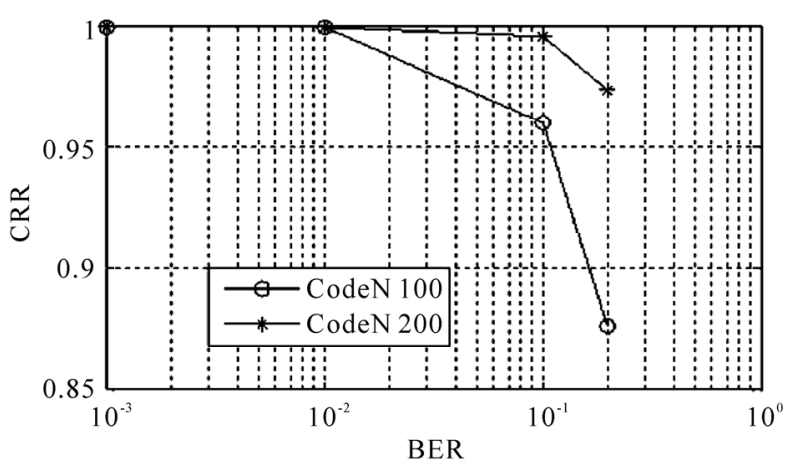

Figure 1. (15,11) code block length recognition capability.

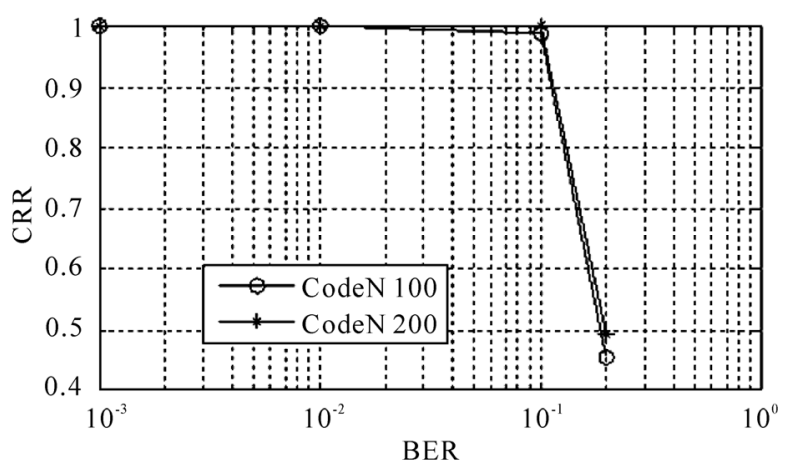

Figure 2. $(15,11)$ code generator polynominal recognition capability.

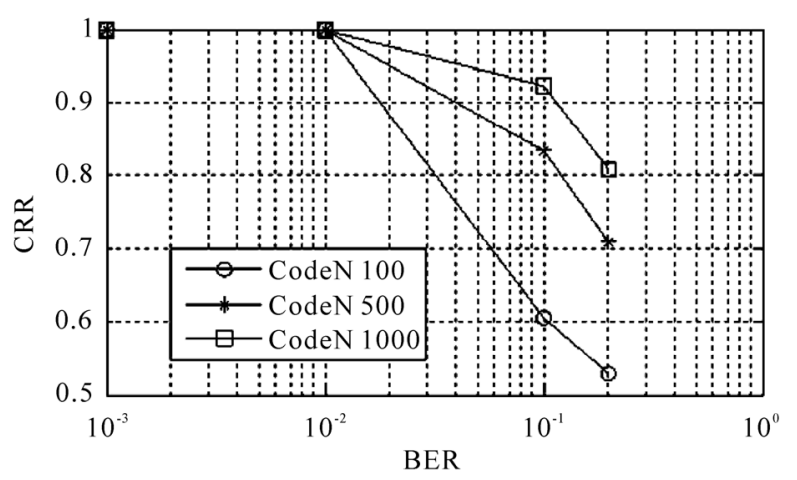

Figure 3. $(21,12)$ code block length recognition capability.

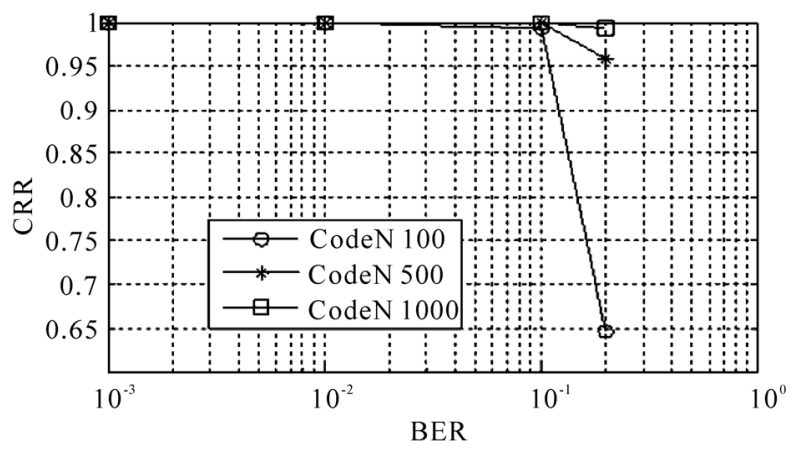

Figure 4. $(21,12)$ code generator polynominal recognition capability. 


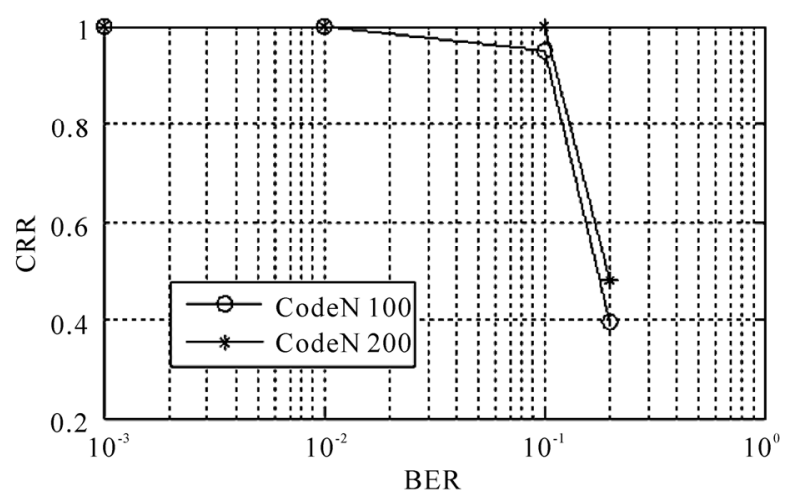

Figure 5. (15,11) code total recognition capability.

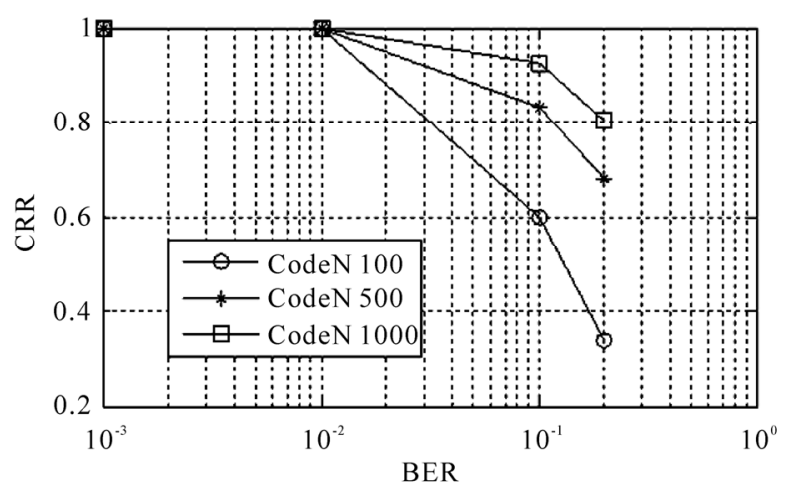

Figure 6. $(21,12)$ code total recognition capability.

Note: In pictures, BER represents "Bit Error Rate", CRR represents "Correct Recognition Rate", and CodeN repesents "Code-words Number".

\subsection{Analysis of Simulation Results}

From the results of the simulation, we know that no matter the recognition capability of the block length, the recognition capability of the generator polynomial, or the total recognition capability, will increase with the number of code words that are used for the recognition process. The recognition capability will also increase with the circular shifting time. Because of the limitation of space, the simulation results are not given here.

Otherwise, we can see that under the condition of the number of code words is the same, the difference between the recognition capability of these two kinds of code words is relatively large. For $(15,11)$ code, when the error code rate is $10 \%, 100$ code words are used, its total recognition capability could reach above $90 \%$. While for $(21,12)$ code, when the error code rate is the same, to reach the same recognition capability, we need 1000 code words. The reason for this difference is various,

1) The code length of $(21,12)$ code is longer than
$(15,11)$ code, then when the code words is the same, through a circular shift, the percentage of satisfying circular feature would be higher than $(15,11)$ code, even if not right blocking.

2) The frame length of $(21,12)$ code is 105 , it has 6 odd factor: $3,5,7,15,1,35$; while the frame length of $(15,11)$ code is 75 , it has 4 odd factor: $3,5,15,25$. Obviously, when the code word number is the same, the more the factor, the worse the recognition capability.

Of course, the recognition capability is also relevant to the structure of the code, $(15,11)$ code is primitive binary $\mathrm{BCH}$ code and $(21,12)$ code is non-primitive binary $\mathrm{BCH}$ code. But we can see from the simulation result, although when the number of code words is the same, the recognition capability of $(21,12)$ code is worse than $(15,11)$ code, but we can increase code words to reach the same recognition capability .

\section{Conclusions}

The blind recognition method of the binary $\mathrm{BCH}$ code proposed in this paper is a statistical recognition method. So the recognition capability is directly related to statistical bit number, the more the statistical bits, the better the recognition capability and the bigger the computation complexity, especially under the condition of long code, which is a drawback of the algorithm proposed in this paper. But when compared to its recognition capability, the big computation complexity is acceptable. Besides, the algorithms in this paper do not involve complicated computations, and it could be readily applied to the hardware processor because of its binary characteristics. So the hardware implementation is easy and the computation can be accelerated.

\section{Acknowledgment}

The work in this paper is supported by the Fund of Science and Technology Development of CAEP. The number is 2009B0403043.

\section{References}

[1] F. H. Wang, Z. T. Huang and Y. Y. Zhou, "A Method for Blind Recognition of Convolution Code Based on Euclidean Algorithm," IEEE International Conference on Wireless Communication Networking and Mobile Computing, Shanghai, 2007, pp. 1414-1417. doi:10.1109/ WICOM.2007.358

[2] P. Z. Lu, L. Shen, Y. Zou and X. Y. Luo, "Blind Recognition of Punctured Convolutional Codes," Science in China, Vol. 48, No. 4, 2005, pp. 484-498. doi: 10.1360/ 03 yf0480 
[3] J. J. Zan and Y. B. Li, "Blind Recognition of Low Code-Rate Binary Linear Block Code," Radio Engineering of China, Vol. 39, No. 1, 2009, pp. 19-24.

[4] J. Liu, N. Xie and X. Y. Zhou, "Blind Recognition Method of RS Coding," Journal of Electronic Science and Technology, Vol. 38, No. 3, March 2009, pp. 363367.

[5] S. Lin and D. J. Costello, "Error Control Coding," 2nd
Edition, Pearson Prentice Hall, Upper Saddle River, 2004, pp. 136-146.

[6] X. Wang, X. M. Wang and B. D. Wei, "An Efficient and Deterministic Algorithm to Determine Irreducible and Primitive Polynomials over Finite Fields," Acta Scientriarum Naturalium Universitatis Sunyatseni, Vol. 48, No. 1, 2009, pp. 6-9. 